\title{
Tuberculum Sellae Meningioma Resection: Technical Nuances on the Frontopterional Approach
}

\author{
Oriela Rustemi $^{1}$ Renato Scienza ${ }^{1}$ Alessandro Della Puppa ${ }^{1}$ \\ ${ }^{1}$ Department of Neurosurgery, Padua University Hospital, \\ Padua, Italy \\ J Neurol Surg B 2018;79(suppl S2):S225-S226. \\ Address for correspondence Oriela Rustemi, MD, Department of \\ Neurosurgery, Padua University Hospital, Azienda Ospedaliera di \\ Padova, via Giustiniani, 2-35128, Padova, Italy \\ (e-mail: orielarustemi@libero.it).
}

\begin{abstract}
Keywords

- tuberculum sellae meningioma

- pterional approach

- cisternal anatomy

- optic canal

Tuberculum sellae meningioma remains a surgical challenge. Deep location of tumor, vascular and nerve encasement, and pituitary stalk involvement are the main technical issues. The frontopterional approach represents a natural, simple, and elegant approach to this area enabling surgeon to have a direct control on all anatomical structures. A 42-year-old woman was referred with a delayed diagnosis of tuberculum sellae meningioma due to the presence of HLA-B27-associated uveitis. She presented with $1 / 10$ visual acuity in the left eye and no right visual function. A left frontopterional craniotomy was performed. Visual function improved postoperatively. The video illustrates the cisternal anatomy via pterional approach.

The link to the video can be found at: https://youtu.be/Hmbf5bt7A64.
\end{abstract}

Conflict of Interest

None.

Funding

None.

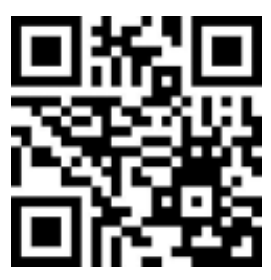

www.thieme.com/skullbasevideos

www.thieme.com/jnlsbvideos received

July 8,2017

accepted

December 12, 2017

published online

January 15, 2018
DOI https://doi.org/

10.1055/s-0037-1620245.

ISSN 2193-6331. (c) 2018 Georg Thieme Verlag KG

Stuttgart · New York
License terms

(ㄷ) (i) $\ominus$ (\$) 

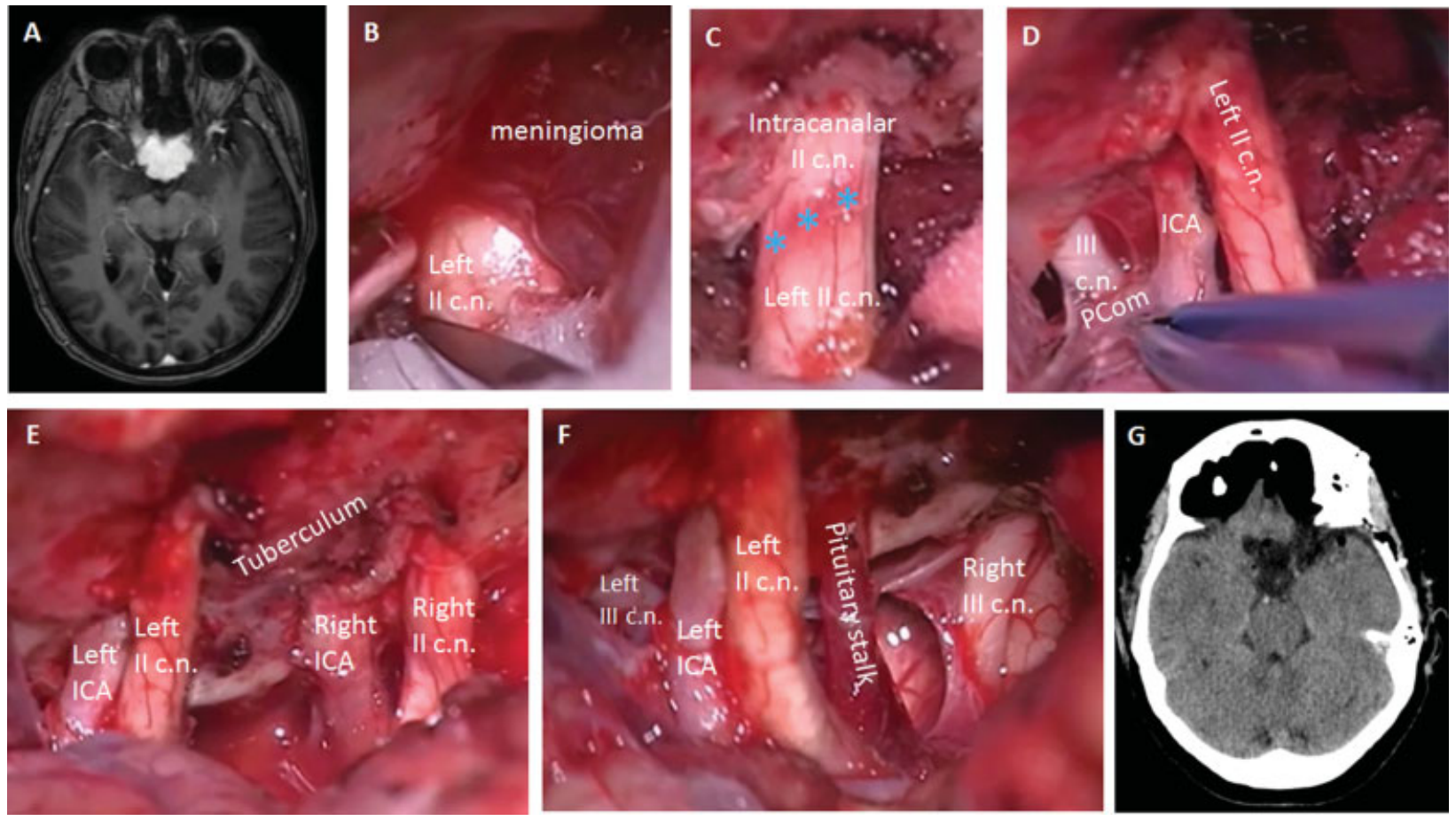

Fig. 1 (A) Preoperative MRI shows a contrast-enhanced tuberculum sellae lesion, suspicious of meningioma. (B) The left optic nerve (II c.n.) is evidenced through a left pterional approach. The II c.n. is compressed by the meningioma. (C) The left optic canal is unroofed and the sign of optic nerve compression is indicated by blue asterisks $\left({ }^{*}\right)$. (D) Intraoperative image after removing the left part of the meningioma showing the left optic nerve, internal carotid artery (ICA) and posterior communicating artery (PCom). (E) Intraoperative image after the removal of the meningioma showing bilateral optic nerves and ICAs, and the tuberculum meningioma's base. (F) Final intraoperative view showing bilateral oculomotor nerve (III C.n.), left II c.n., left ICA, and the conserved pituitary stalk. (G) Postoperative CT shows the complete removal of the meningioma. $\mathrm{CT}$, computed tomography; MRI, magnetic resonance imaging. 